Bangladesh J. Plant Taxon. 14(1): 47-66, 2007 (June)

\title{
BRYOPHYTE FLORA OF GREATER MYMENSINGH DISTRICT OF BANGLADESH - CLASS : BRYOPSIDA
}

\author{
KhURShida BANU-FatTAH ${ }^{1}$ AND SUJAN KumER SARKER \\ Department of Botany, Govt. Ananda Mohan College, \\ College Road, Mymensingh, Bangladesh
}

Key words: Bryophytes, Bryopsida, Mosses, Mymensingh District, Bangladesh

\begin{abstract}
The Greater Mymensingh District, particularly the hilly areas are rich in bryophyte flora. The present paper on Bryopsida is based upon primary as well as secondary data collection and includes an account of 51 species under 35 genera, 21 families and nine orders. A short description of each species with locations, dates of collections and name of collectors are provided.
\end{abstract}

\section{Introduction}

A reasonably good amount of works have been done on mosses of Bangladesh (Banu 1991, Khatun 2002 and references therein). However, our knowledge on districts or zonal distribution is very scanty. Tixier (1967) collected and reported many mosses from Chittagong and Cox's Bazar of Bangladesh, but none from Greater Mymensingh District, which currently includes six administrative districts, namely Jamalpur, Kishoreganj, Mymensingh, Netrokona, Sherpur and Tangail. Banu (1991) for the first time gave a district-wise distribution of mosses, but her work was confined only to Acrocarpous mosses. Banu-Fattah (1998) dealt with bryophytic flora of Chittagong Zone where a number of hepatics and mosses were reported, but the areas covered were limited to a few sites only. So we do not have a complete record of bryophytes as a whole from any district or zone.

In the present paper, an attempt has been made for the first time to prepare a comprehensive list of mosses under the class Bryopsida of the Greater Mymensingh District. Here the authors have covered many localities of the present administrative districts of Greater Mymensingh by collecting a large number of bryophytes including mosses. All the previous reports on Bryopsida from the Mymensingh region which have been published so far were also consulted while preparing the list.

\section{Materials and Methods}

The areas covered in this study constitute the Greater Mymensingh District (latitude $24^{\circ} 05^{\prime}$ and $25^{\circ} 36^{\prime}$ and longitude $89^{\circ} 70^{\prime}$ and $91^{\circ} 20^{\prime}$ ) of Dhaka Division of Bangladesh which at present includes six administrative districts. This area is on the centre of the

${ }^{1}$ Corresponding author. Present address: C/o Prof. Quazi Abdul Fattah, Department of Botany, University of Dhaka, Dhaka 1000, Bangladesh. E-mail: botany@univdhaka.edu 
northern side of Bangladesh. Northern part of this zone have hills and hillocks which is an extension of Garo Hills of Himalayan-Hindukush mountain range of India, the other part of this region is mostly low-lying plains. Some part of this region is above $10 \mathrm{~m}$ and the rest is about $3 \mathrm{~m}$ above sea level. The land is intersected with a large number of rivers, streams and canals of which the River Brahmaputra is the most important one. Temperature of this zone usually ranges from $11.6^{\circ} \mathrm{C}$ in January to $32.7^{\circ} \mathrm{C}$ in July. Annual rainfall is moderate, being $232 \mathrm{~cm}$ and humidity varies from 62-95\%. The central part and also some scattered strips of this zone have natural forests.

This report is mainly based upon fresh materials collected from different localities under the Greater Mymensingh District area. Most of the bryophytes collected were worked out and identified and all the specimens collected are preserved in the Bryology Herbarium, Department of Botany, Govt. Ananda Mohan College, Mymensingh. In addition, the occurrence of many species is based on previously published documents. Banu-Fattah and Hadiuzzaman in a series of publications (1994, 1995, 1996a,b,c, 1998a,b,c, 2003a,b, 2004, 2006a,b) on the Acrocarpous mosses of Bangladesh described several species from the families Polytrichaceae, Ditrichaceae, Dicranaceae, Leucobryaceae, Calymperaceae, Fissidentaceae, Pottiaceae, Funariaceae, Splachnaceae, Bryaceae and Bartramiaceae from the studied region. Khatun (2002) reported many Pleurocarpous mosses from this region, but only those mosses are included here which have been published so far (Khatun and Hadiuzzaman 1994, 1995, 2003, 2004, 2005, 2006, 2007a,b). The specimens used by Banu (1991) and Khatun (2002) are preserved in the Bryology Herbarium, Department of Botany, University of Dhaka (DUBH).

The classification followed in this enumeration is that of the system used by Gangulee (1969-1980). A short description of each species with habitats, locations, dates and collector's name(s) are provided. Basionyms are also provided where appropriate/available.

\section{Results}

The present study comprises an account of 51 species under 35 genera, 21 families and nine orders. Although this study is not very extensive, it shows that the Greater Mymensingh District is a good abode of mosses, particularly, the Acrocarpous mosses (Banu 1991).

Hadiuzzaman (1984) for the first time described Calymperes tenerum Hedw. from Tangail of the Greater Mymensingh. Over the years, a number of interesting findings have been attained regarding the occurrence of mosses in Greater Mymensingh. For example, Banu-Fattah and Hadiuzzaman (1997) described a new species Splachnobryum schofieldii Banu-Fattah et Syed from Tangail. Later on, Banu-Fattah and Lal (1998) reported the occurrence of a very rare monotypic moss, Pleuridiella colei Robinson, previously thought to be endemic in India, collected from Kishoreganj, Mymensingh and 
Tangail Districts. Moreover, the three most common mosses of Bangladesh, namely, Semibarbula orientalis (Web.) Wijk. \& Marg., Hyophila involuta (Hook.) Jaeg. and Bryum apiculatum Schwaegr. are also very common in this region (Banu-Fattah and Hadiuzzaman 1993). Recently, Funaria hygrometrica Hedw., a well-known moss of the world, was collected only from Tangail District (Banu-Fattah 2005).

It has been observed that the mosses are adapted to a wide range of habitats, but most of the Acrocarpous mosses are either terrestrial or lithophytes prefering shady, moist places of the plains or slope of hills. Most of the Pleurocarpous mosses and the species of the families Leucobryaceae and Calymperaceae of Acrocarpous mosses are epiphytes. Some species, specially, Trematodon longicollis Michx., are common in Mymensingh and grow by the sides of rivers and ponds.

Robinson (1964) reported many mosses from Assam region of India, adjacent to this area, and those mosses along with many others are expected to be present here. An extensive and intensive collection will surely add more names to the moss flora of Greater Mymensingh District. More studies are needed to complete the zonal or districtwise distribution of the mosses of this region.

A brief account with relevant information on all the 51 species found in the region is given in the following section.

\section{Taxonomic enumeration}

Class : Bryopsida; Order : Polytrichales; Family : Polytrichaceae

Genus : Pogonatum Palisot de Beauvois, in Mag. Enc. 5: 329 (1804).

1. Pogonatum flexicaule Mitt., in Musc. Ind. Or. 152 (1859).

Plants long, slender, dark-green; lamellae numerous covering almost entire ventral surface, 2-3 celled high at costa; sharply dentate margin; costa spinose-papillose on back, ending in an apicule. Grows on sandy soil, slope of hills and waterfall.

Specimens examined: Jamalpur: Lawachapra, on slope of hill, S.K. Sarker, 2.2.1999; Mymensingh: Haluaghat, on slope of hill, Almas Uddin Hawlader, 30.9.1988.

2. P. hexagonum Mitt., in Musc. Ind. Or. 151 (1859).

Plants dioicous, sturdy, medium-sized, reddish; lamellae numerous, covering almost entire ventral surface; costa promiment, percurrent, spinose; capsule hexagonal; peristome teeth 32; calyptra felty. Grows on sandy soil.

Specimen examined: Mymensingh: Panihata, Haluaghat, Almas Uddin Hawlader, 26.12.1988. 


\section{Order : Dicranales; Family : Ditrichaceae \\ Genus : Garckea C. Muell., Bot. Zeit. 3: 865 (1845)}

3. Garckea phascoides (Hook.) C. Muell., Bot. Zeit. 3: 865 (1845).

Dicranum phascoides Hook., Misc. Bot. 1: 39 (1829).

Plants dioicous; yellowish-green; stem long with sparse leaves all along but crowded in comal tuft at tips; sporophyte hidden within perichaetial leaves; seta very short with vaginula; peristome teeth 16, papillose; calyptra scabrous. Grows on dry damp soil, commonly found in hilly areas.

Specimens examined: Jamalpur: Lawachapra, S.K. Sarker, 25.1.1993; Mymensingh: Haluaghat, S.K. Sarker, 3.2.1988; Sherpur: Zhinaigati, Gazni, Ainul Haque and Abu Bakar Siddique, 5.1.1988; near Sherpur Town, Ainul Haque and Abu Bakar Siddique, 20.1.1988; Rungtia Reserve Forest, Almas Uddin Hawlader, 13.12.1988; Tangail: Rasulpur, Modhupur Forest, Almas Uddin Hawlader, 4.11.1987 and 13.1.1988; Ainul Haque and Abu Bakar Siddique, 10.1.1988.

Genus : Ditrichum Hamp., Flora 50: 18 (1867).

4. Ditrichum difficile (Dub.) Fleisch., Musci Fl. Buitenzorg. 1: 300.50 (1900-1902).

Plants yellowish-green, up to $1 \mathrm{~cm}$ high; leaves falcate with broad, ovate sheathing base and abruptly narrowing flexuose, canaliculated subula; laminal cells rectangular to linear, vermicular; leaf margin bistratose in upper part. Grows on sandy soil.

Specimen examined: Sherpur: near Sherpur Town, Almas Uddin Hawlader, 16.2.1990.

Genus : Pleuridiella Robinson, J. Hattori Bot. Lab. 27: 125 (1964).

5. Pleuridiella colei Robinson, J. Hattori Bot. Lab. 27: 125 (1964).

Plants paroicous, very small, 2.5-4.5 mm with leaves; leaf margin narrowly revolute, serrulate all along, cells highly mamillose; capsule immersed, spherical, cleistocarpic; peristome teeth, annulus, operculum absent. Grows on damp soil.

Specimens examined: Kishoreganj: near Kishoreganj Town, Md. Abul Hassan, 7.2.1987; Mymensingh: Womens’ T.T. College, K.B. Fattah, 2.2.1987 and 5.11.1996; by the side of Brahmaputra River, near Circuit House, K.B. Fattah and S.K. Sarker, 5.11.1996: NAPE campus, K.B. Fattah and S.K. Sarker, 5.11.1996; Tangail: near Tangail Town, Md. Kamruzzaman, 14.2.1988. 


\section{Family : Dicranaceae}

Genus: Dicranella (C. Muell.) Schimp., Caroll. Bry. Eur.: 13 (1856).

Aongstroemia C. Muell., Syn. 1: 430 (1848).

6. Dicranella amplexans (Mitt.) Jaeg., Ber. S. Gall. Naturw. Ges. 1870-71: 376 (1872).

Leptotrichum amplexans Mitt., in Musc. Ind. Or. 9 (1859).

Plants dioicous; in dense tuft; stem reddish, unbranched, upper leaves longer, crowded at apex; leaf margin smooth; costa percurrent; seta with vaginula; capsule inclined; peristome teeth 16, dicranate. Grows on sandy soil.

Specimen examined: Sherpur: in and around Sherpur Town, Almas Uddin Hawlader, 16.2.1990.

Genus : Trematodon Michx., Fl. Amer. Bor. 2: 289 (1803).

7. Trematodon longicollis Michx., Fl. Amer. Bor. 2: 289 (1803).

Plants autoicous; densely gregarious, yellow-green; stem very short, c $1.5 \mathrm{~mm}$ with leaves; costa prominent; sporophyte with long, curved, yellowish-brown capsule with long spongy apophysis with distinct struma at base; peristome teeth 16, irregularly perforated or splitted; operculum conic-rostrate; calyptra cucullate; spores opaque, papillose. Grows on soil, commonly by the side of waterfalls, streams and rivers. Common in Mymensingh District.

Specimens examined: Jamalpur: near Jamalpur Town, Anamul Haque, 12.2.1990 and 10.3.1993; Mymensingh: by the side of Brahmaputra river, K.B. Fattah, 15.3.1987; Ainul Haque and Abu Bakar Siddique, 11.2.1988; Shaheb Park, K.B. Fattah and S.K. Sarker 8.12.1992; Sherpur: Gazni, Ainul Haque and A.B. Siddique, 2.1.1988; Rungtia Reserve Forest, Almas Uddin Hawlader, 13.12.1988; near Sherpur Town, K.B. Fattah and S.K. Sarker, 5.12.1990.

\section{Family : Leucobryaceae}

Genus : Leucophanes Besch. in Brid., Bryol. Univ. 1: 763 (1826).

8. Leucophanes glaucescens C. Muell. ex Fleisch., Musci Fl. Buitenz. 1: 178 (1904).

Plants dioicous; whitish-green; leaf margin bordered all along with a narrow band of very incrassate cells; costa broad, completely covers upper 2/3- 2/5 length of lamina, one layer of chlorocyst cells, 4-sided; capsule orange-red; peristome teeth 16, papillose, deep inserted; multicellular gemmae present at the apex of leaves on costa. Grows on bark of trees.

Specimens examined: Mymensingh: Shaheb Park, K.B. Fattah and S.K. Sarker, 10.9.1987; Gulkibari, K.B. Fattah and S.K. Sarker, 5.8.1993. 
Genus : Octoblepharum Hedw., Sp. Musc. 50 (1801).

9. Octoblepharum albidum Hedw., Sp. Musc. 50 (1801).

Plants autoicous; in dense cushion; leaves whitish-green, flat, thick, lingulate, loosely attached to stem, chlorocysts 3-sided; costa broad, occupying half of leaf base and entire blade; peristome teeth in eight pairs; operculum conic-rostrate; spores finely papillose; new plants grow from apex of leaves. Grows on bark of trees.

Specimens examined: Mymensingh: Boroikandi, Phulpur, S.K. Sarker, 5.7.1996; Shaheb Park, K.B. Fattah and S.K. Sarker, 25.11.2000; Sherpur: Zhinaigati, Gazni, Abu Bakar Siddique, 9.9.1987; Tangail: near Kumudini Girl's College, K.B. Fattah, Bilkis Banu and Badrunnesa, 12.12.1990.

\section{Order : Fissidentales; Family : Fissidentaceae \\ Genus : Fissidens Hedw., Sp. Musc. 152 (1801).}

10. Fissidens bryoides Hedw., Sp. Musc. 153 (1801).

Plant gregarious; shoot with 8-10 pairs of leaves; sheathing lamini equal; limbidium all along leaf; costa percurrent or slightly excurrent; laminal cells hexagonal, smooth. Grows on damp soil and old bricks.

Specimens examined: Mymensingh: Phulpur Town, K.B. Fattah, Jashim Uddin and S.K. Sarker, 5.10.1989 and 6.11.2000; Tangail: Nagarbari, Md. Shafiuddin Mia, 21.6.1989.

11. F. geminiflorus Doz. et Molk., Pl. Jhungh. 3b. (1854).

Plants dioicous; leaves up to 28 pairs; sheathing lamini $1 / 2-2 / 3$ of whole leaf, excurrent; laminal cells quadrate to round, multipapillose. Grows on damp soil and old bricks.

Specimen examined: Mymensingh: College Road, K.B. Fattah, 9.8.1987.

12. F. laxus Sull. et Lesq., Proc. Am. Arc. Art. Sc. 4: 276 (1859).

Plants dioicous; densely gregarious, yellow- green; leaves up to 12 pairs; sheathing lamini c $1 / 2$ of whole leaf, unequal; margin crenulated to serrulate with papillae; costa percurrent or short- excurrent; laminal cells big, hexagonal, multipapillose. Grows on red, sandy soil and moist bricks.

Specimen examined : Tangail: Modhupur, K.B. Fattah, 12.2.1990. 
13. F. splachnobryoides Broth. in Schum. et Lauterb., Fl. Deutsch. Schutz. Suedesee 81, 1900.

Plants yellowish to light-green; shoot with about 16 pairs of leaves; limbidium of 1-3 rows of elongated cartilagenous cells all around leaf; costa ends far below apex; laminal cells smooth, transparent, thin-walled; multicellular gemmae present at the axil of leaves, Grows on damp soil.

Specimens examined: Mymensingh: Botanical garden, Bangladesh Agriculture University, K.B. Fattah and Quazi Abdul Fattah, 1.10.1987; Ananda Mohan College, K.B. Fattah and Anamul Haque, 1.10.1987; BARC campus, K.B. Fattah and S.K. Sarker, 19.11.1996.

14. F. sylvaticus Griff., Cal. J. Nat. Hist. 2: 507 (1842).

Plants gregarious; leaves 15-18 pairs; glandular protuberances of 5-6 cells present along stem; sheathing lamini 2/3 of whole leaf, closed; margin crenulated; costa strong, percurrent or slightly excurrent in a mucro; laminal cells hexagonal. Grows on soil, usually in hilly areas.

Specimen examined: Mymensingh: Panihata, Haluaghat, Almas Uddin Hawlader, 26.12.1998.

15. F. zollingeri Mont., Ann. Sci. Nat. Ser. 3, 4: 114 (1845).

Plants synoicous; caespitose, bright-green, radiculose; leaves 5-8 pairs, sheathing lamini about half of whole leaf length, equal; limbidium borders whole leaf length; costa slightly excurrent; seta geniculate, flexuose, orange in colour; peristome dicranate, orange; spores smooth. Grows on sandy soil, often on slope of hills.

Specimens examined: Mymensingh: College Road, K.B. Fattah and A. Haque 8.10.1987; Teachers' Training College, S.K. Sarker, 25.8.1999; Haluaghat, S.K. Sarker, 26.8.1999; Sherpur: Gazni, K.B. Fattah and S.K. Sarker, 9.9.1987; Tangail: Modhupur, K.B. Fattah, Bilkis Banu and Badrun Nessa, 12.2.1990.

Order : Syrrhopodontales; Family : Calymperaceae

Genus : Calymperes Schwaegr. in Web., Tab. Exh. Calyp. Operc. Gen. 2 (1813).

16. Calymperes tenerum C. Muell., Linnaea 37: 174 (1871).

Plants small, with short stem and rosette of leaves; leaf apex obtuse; margin entire; cancellinae distinct, rectangular with flat top, $1 / 3$ to $1 / 6$ of whole leaf; tenioli absent; gemmiferous leaves bear cluster of club to spindle-shaped gemmae at the apex of excurrent costa. Grows on bark of trees.

Specimens examined: Tangail : Gorai, M.A. Quader, 2.2.1975 and 3.2.1977. 
Order : Pottiales; Family : Pottiaceae

Genus : Hyophila Brid., Bryol. Univ. 1: 760 (1827).

17. Hyophila comosa Dix. et Varde, Arch. Bot. 1: 166 (1927).

Plants densely tufted; leaves strongly inrolled and curled when dry, oblong-lingulate to spathulate, apex obtuse to rounded; costa strong percurrent; upper laminal cells multipapillose; gemmae abundant at the axil of leaves. Grows on old, damp bricks.

Specimens examined: Mymensingh: Haluaghat, Jashim Uddin, 10.9.1989; Netrokona: in and around Netrokona Town, Anamul Haque, 12.9.1988.

18. H. involuta (Hook.) Jaeg., Ber. S. Gall. Naturw. Ges. 1871-72: 356 (1873).

Gymnostomum involutum Hook., Musc. Exot. 2: 154 (1819).

Plants dioicous; in dense tufts; leaf margin denticulate at apex, strongly involute when dry; costa strong, red-brown; capsule long, cylindrical; peristome absent; calyptra spirally twisted, cucullate; spores smooth; multicellular gemmae abundant on branched filaments at the axil of leaves. Very common species. Grows mostly on damp, old, concrete walls, drains, bricks and stones.

Specimens examined: Mymensingh: Torun Hostel, Ananda Mohan College, K.B. Fattah and Anamul Haque, 10.4.1987; Botanical Garden, Bangladesh Agriculture University, K.B. Fattah and Quazi Abdul Fattah, 28.9.1987; Masjid road, Kachijhuli, K.B. Fattah, 14.10.1987; Women Teachers' Training College campus, K.B. Fattah and Sayeeda Sarwar, 16.1.1988, 28.4.1989 and 26.8.1997; Phulpur Town, Jashim Uddin, 5.10.1989; Iswarganj, Shahabuddin, 20.12.1989, S.K. Sarker, 26.8.1997; Tangail: Kagmari College, K.B. Fattah and Farida Begum, 3.3.1987.

Genus: Hydrogonium (C. Muell.) Jaeg., Ber. S. Gall. Naturw. Ges. 1877-78: 405 (1880). Section Hydrogonium of Trichostomum C. Muell., Linnaea 40: 297 (1876).

19. Hydrogonium arcuatum (Griff.) Wijk. \& Marg., Taxon 7: 289 (1958).

Barbula arcuata Griff., Cal. J. Nat. Hist. 2: 491 (1842).

Plants dioicous; yellowish-green, tubers present on rhizoid; all laminal cells unistratose, smooth; capsule short-cylindrical, peristome teeth filiform, highly papillose; operculum conic-rostrate; calyptra cucullate; oval to star-shaped gemmae present at axil of leaves. Grows on damp soil.

Specimen examined: Tangail: Horticulture Farm, Md. Kamruzzaman, 10.10.1987. 
Genus : Semibarbula Herz. ex Hilp., Beih. Bot. Centralbl. 50(2): 626 (1933).

20. Semibarbula orientalis (Web.) Wijk. \& Marg., Taxon 8: 75 (1959).

Trichostomum orientale Web., Arch. Syst. Nat. 1: 129 (1804).

Plants dioicous; slender, unbranched; leaves short, oblong with distinct plica on each side of costa; margin papillose; apex obtuse; costa strong with hyaline apicule or percurrent, very rough, corrugated with papillae, upper laminal cells multipapillose, obscure; peristome teeth 16, erect, highly papillose; multicellular gemmae abundant, apical or axillary. Grows on damp bricks, cement walls and stones. Very common.

Specimens examined: Kishoreganj: Kishoreganj Town, Md. Abul Hassan, 2.2.1987; Mymensingh: Station Road, K.B. Fattah and A. Haque 13.3.1987; Ananda Mohan College, Quazi Abdul Fattah, 28.9.1987; Women Teachers' Training College, K.B. Fattah and Syeeda Sarwar, 28.9.1987, 16.1.1988 and 26.8.1997; Kadirpur, K.B. Fattah, 17.8.1987; Masjid Road, Kachijhuli, K.B. Fattah and A. Haque, 14.11.1987; Ishwarganj, K.B. Fattah and Shehab Uddin, 20.12.1988; Shaheb Park, S.K. Sarker, 28.9.1989 and 25.11.2000; Botanical garden, Bangladesh Agriculture University, K.B. Fattah and Quazi Abdul Fattah, 28.9.1987 and 26.8.1997; Netrokona: Netrokona Town, Anamul Haque, 12.9.1988; Sherpur: Rungtia Reserve Forest, Almas Uddin Hawlader, 13.12.1988; Tangail: Kagmari College, Md. Kamruzzaman, 3.3.1987; Nagarpur, Md. Shafiuddin Mia, 21.6.1989.

Genus : Barbula Hedw., Sp. Musc., 115 (1801).

21. Barbula marginatula C. Muell. ex Gang., Nova Hedwigia 12: 424 (1966).

Plants dioicous; densely tufted, yellowish-green; leaves with a very distinct hyaline margin of 2-3 rows of elongated papillose cells; costa strong; middle and upper laminal cells incrassate, densely papillose. Grows on sandy soil and walls.

Specimen examined: Mymensingh: Haluaghat, Jashim Uddin, 10.9.1989.

Order : Funariales; Family : Splachnaceae

Genus : Gymnostomiella Fleisch., Musci Fl. Buitenzorg 1: 309 (1904).

22. Gymnostomiella vernicosa (Hook.) Fleisch., Musci Fl. Buitenzorg 1: 310 (1904).

Gymnostomum vernicosum Hook., Icon. Pl. Rar. 1: (1836).

Plants in dense compact tuft or loose and scattered, small, delicate; leaves lax and distant below, crowded at apex, upper leaves broad, obovate with rounded apex; margin very rough above; costa extends up to $2 / 3$ of leaf; laminal cells thin, upper cells multipapillose; gemmae present at the axil of leaves. Grows on damp walls, bricks, and drains. 
Specimens examined: Mymensingh: Muktagachha Rajbari, K.B. Fattah and S.K. Sarker, 3.12.1987; Shaheb Park, K.B. Fattah and S.K. Sarker, 28.9.1989; Sherpur: Rungtia Reserve Forest, Almas Uddin Hawlader, 13.12.1988; Tangail: Nagarpur, Md. Shafiuddin, 21.6.1989.

Genus : Splachnobryum C. Muell., Verh. Zool. Bot. Ges. Wien. 19: 503 (1869).

23. Splachnobryum schofieldii Banu-Fattah et Syed, Bangladesh J. Bot. 26(1): 61-64 (1997).

Plants dioicous, slender, soft, unbranched; leaves broad, obovate to sub-orbicular with obtuse to rounded apex; laminal cells very thin-walled; costa ends much below apex. Grows on damp soil.

Specimen examined: Tangail: Nagarpur, Md. Shafiuddin Mia, 21.6.1989.

Family : Funariaceae

Genus : Funaria Hedw., Sp. Musc., 172 (1801).

24. Funaria hygrometrica Hedw., Sp. Musc., 172 (1801).

Autoicous; stem short with rosette of leaves at apex; laminal cells thin-walled, smooth, large; seta long, slender, arcuate at top, reddish; capsule pyriform, asymmetric with narrow oblique mouth; apophysis distinct; peristome typical diplolepideous, epicranoid; plano-concave; operculum without apiculus; calyptra cucullate. Grows on damp soil. Very rare.

Specimen examined: Tangail: Ghatail, K.B. Fattah and S.K. Sarker, 5.12.1992.

Genus: Entosthodon Schwaegr., Sp. Musc. Suppl. 2: 44 (1823).

25. Entosthodon nutans Mitt., Musc. Ind. Or. 55 (1859).

Plants scattered, small, up to 2-3 mm with rosette of leaves at apex; laminal cells very lax, smooth, thin-walled; capsule turbinate without apophysis; no peristome; operculum flat, without apiculus; spores fine papillose. Grows on damp soil.

Specimens examined: Kishoreganj: in and around Kishoreganj Town, Md. Abul Hassan, 2.2.1987; Mymensingh: Near Ananda Mohan College, S. Hadiuzzaman, 15.3.1989; Netrokona: Mohanganj, Abdul Momen, 21.12.1990.

Genus : Physcomitrium (Brid.) Fuernr., Flora 13: 9 (1829).

Physcomitrium (of Gymnostomum) Brid., in Bryol. Univ. 1: 97 (1826).

26. Physcomitrium cyathicarpum Mitt., Musci Ind. Or. 54 (1859).

Plants paroicous; tufted or scattered; stem very short with crowded leaves at apex; laminal cells thin-walled, large; seta very short; capsule globose, immersed; no 
peristome; operculum apiculate; spores densely papillose. Grows on damp soil, mostly in gardens and sides of rivers and ponds.

Specimens examined: Mymensingh: Shaheb Park, near Brahmaputra River, K.B. Fattah and Ainul Haque, 3.2.1988; College Road, near Ananda Mohan College, S. Hadiuzzaman, 15.3.1989. Gaffargaon, B.C. Shaha, 15.11.1989.

27. P. eurystomum Sendtn., Denkschr. Bayer Bot. Ges. Regensb. 3: 142 (1841).

Plants dioicous; stem short with rosette of leaves; leaf margin sharply serrulate above; laminal cells thin-walled, bordered by one row of elongated cells; capsule pyriform with apophysis; calyptra lobed; spores papillose. Grows on damp soil, specially on gardens and by sides of rivers and ponds.

Specimens examined: Mymensingh: Town Park, by the side of Brahmaputra River, K.B. Fattah and A. Haque, 3.2.1988 and 10.9.1989; Gaffargaon Town, B.C. Shaha, 15.11.1989; Netrokona: Mohanganj, Abdul Momen, 21.12.1990; Tangail: near Kumudini Girl’s College, K.B. Fattah, 12.2.1990.

28. P. pulchellum (Griff.) Mitt., Musci Ind. Or. 54 (1859).

Gymnostomum pulchellum Griff., Cal. J. Nat. Hist. 2: 478 (1842).

Plants dioicous; densely tufted; stem radiculose; leaves crowded above; margin entire; costa short-excurrent; laminal cells thin-walled, smooth; leaf bordered by one layer of elongated, vermicular cells; capsule turbinate to pyriform with short apophysis, mouth narrower than urn; spores warty papillose. Grows on damp soil, specially in gardens.

Specimens examined: Mymensingh: Botanical Garden, Bangladesh Agriculture University, K.B. Fattah and Quazi Abdul Fattah, 10.10.1987 and 14.1.1989.

\section{Order : Eubryales; Family : Bryaceae}

Genus : Bryum Hedw., Sp. Musc., 178, 1801. emend. Schimp., Syn. ed. 1. 1860.

29. Bryum apiculatum Schwaegr. in Ichen, Buck. W.R., Atlantic Forest Remnant. Pernambuco, Brazil J. Bryology 24: 251-252 (1990).

Plants in dense tufts, bright green, lustrous; leaves ovate lanceolate, concave, carinate; margin entire; costa percurrent; laminal cells rhomboid; numerous single and clustered multicellular gemmae present on stems, branches also on rhizoids. Grows on soil, specially on plant pots, garden beds and also on damp bricks, walls, and roofs.

Specimens examined: Kishoreganj: In and around Town, K.B. Fattah, 28.2.1990; Mymensingh: Ananda Mohan College, Quazi Abdul Fattah, 28.9.1987, S. Hadiuzzaman, 15.3.1989; Botanical Garden, Bangladesh Agriculture University, Quazi Abdul Fattah 
and K.B. Fattah, 1.10.1987; Sultana Rezia Hall, Bangladesh Agriculture University, K.B. Fattah, 19.1.1989 and 15.9.1989; Circuit House Compound, Quazi Abdul Fattah and K.B. Fattah, 1.10.1987; Phulpur Town, Jashim Uddin, 5.10.1989; Gaffargaon Town, B.C. Shaha, 15.11.1989; Ishwarganj, Md. Shehab Uddin, 20.12.1989; Tangail: near Kumudini Girl's College, Md. Kamruzzaman, 21.3.1988.

Family : Bartramiaceae

Genus : Philonotis Brid., Bryol. Univ. 2: 15 (1827).

30. Philonotis angusta Mitt., Musc. Ind. Or. 61 (1859).

Plants dioicous; robust, densely tufted, shoot long with whorl of innovations; stem tomentose; leaves with broader base, long acuminate apex and serrate margin; capsule sub- globose to ovoid; slightly asymmetric, mouth small. Grows on soil, usually in hills.

Specimen examined: Sherpur: Gazni, Zhinaigati, Almas Uddin Hawlader, 16.2.1990.

31. P. falcata (Hook.) Mitt., Musci Ind. Or. 62 (1859).

Bartramia falcata Hook., Trans. Linn. Soc. Lond. 9: 317 (1808).

Plants dioicous; fairly robust, interwoven with dark brown silky tomenta below; shoot with a whorl of innovations; leaves with percurrent or short-excurrent costa and acuminate apex. Grows on damp bricks, walls, roofs.

Specimen examined: Mymensingh: Ananda Mohan College, Torun Hostel, K.B. Fattah and Anamul Haque, 10.4.1987.

32. P. hastata (Dub.) Wijk. \& Marg., Taxon 8: 74 (1859).

Hypnum hastatum Dub. In Moritzi, Syst. Verz. Zoll. Pfl. 132 (1846).

Plants dioicous; dense, forming mats, very slender, soft, bright green above, loosely attached to the substratum; stem highly radiculose; leaves lax, thin, lingulate with blunt, obtuse apex; costa ending much below apex. Grows on damp brick walls, drains and on nearby soil.

Specimens examined: Mymensingh: Bangladesh Agriculture University, Botanical Garden, Quazi Abdul Fattah and K.B. Fattah, 1.10.1987; Sultana Rezia Hall, K.B. Fattah, 19.1.1989; Circuit House Compound, Quazi Abdul Fattah and K.B. Fattah, 19.1.1989; Phulpur Town, Jashim Uddin, 5.10.1989; Ishwarganj, Md. Shehab Uddin, 20.10.1989; Tangail : in and around Tangail Town, Md. Quamruzzaman, 21.3.1988. 
Order: Isobryales; Family : Erpodiaceae

Genus : Erpodium (Brid.) Brid., in Reichenb., Consp. 32 (1828). Anoectangium subgen, Erpodium Brid., in Bryol. Univ., 2: 167 (1827).

33. Erpodium mangiferae C. Muell., Linnaea 37: 178 (1872).

Plants small with branches, radiculose; monomorphic; leaves in several rows, ovate, concave, short-acuminate; no costa; all leaf cells with primordial utricle, cells not papillose; alar cells rhomboid. Grows on bark of trees.

Specimens examined: Jamalpur: Nandina, Fazar Ali, 3.2.1998; Madarganj, Mokhlesur Rahman, 21.7.1999. Kishoreganj: Katiadi, Bijoy Kumar Modak, 2.5.1998; Mymensingh: By the side of Brahmaputra River, Rafiqul Islam, 30.9.1987; Phulpur, Jashim Sheikh, 6.6.1996; Shombhuganj, Abu Naser Md. Abdullah, 16.7.1997; Trishal, Shahnoor Hossain, 16.7.1997; Bhaluka, Shahnoor Hossain, 16.7.1997; Sherpur: Nalitabari, A.S.M. Asadur Rahman, 15.12.1998; Tangail: Akurtakur Para, S. Hadiuzzaman, 5.12.1996; Mirzapur, Hamida Khatun, 2.10.1991; Karatia, Hamida Khatun, 2.12.1994; Ghatail, Hamida Khatun, 20.12.1990; Modhupur Forest, Farida Rahman, 21.8.1996; Sagardighi, Jamshed Ali, 21.3.2000.

\section{Family : Pterobryaceae}

Genus : Pterobryopsis Fleisch., in Hedwigia 45: 56 (1905).

34. Pterobryopsis auriculata Dix., J. Bombay Nat. Hist. Soc. 39: 782 (1937).

Plants robust, pinnately branched, leaves concave, ovate-cochliariform, cucullate at apex, margin involute in upper half, base with prominent auricle, costa single, cells nonpapillose but porous, alar not conspicuous, gemmae abundant. Grows on bark of trees.

Specimen examined: Tangail: Mirzapur, Nazmul Islam, 19.2.1991.

Family : Neckeraceae

Genus : Neckeropsis Reichdt., in Reise Oesterr. Freg.

Novara Bot. 1(3): 181 (1870).

35. Neckeropsis exserta (Schwaegr.) Broth., Nat. Pfl. ed. 2 11: 188 (1925).

Neckera exserta Hook. ex Schwaegr. Sp. Musc. Suppl. 3 (1): 244 (1828).

Plants long, loose, soft, glossy, irregularly branched; leaves large, truncate, transversely undulate; costa single, ending near apex; laminal cells smooth, incrassate; capsule shortly exerted; peristome neckeroid. Grows on bark of trees.

Specimens examined: Kishoreganj: Karimganj, Shafiqul Islam, 17.9.1998; Nikli, D.M. Kader, 13.11.1998; Mymensingh: Haluaghat, Abdul Awal, 3.7.1998; Tangail: Mirzapur, Nazmul Islam, 3.7.1999; Modhupur Forest, Rajib Kumar Shaha, 2.6.2000. 
36. N. submarginata Card. ex Touw., Blumea 11: 417 (1962).

Plants yellow-green, glossy; branches rigid; leaves complanate, tetrastichous, lingulate, slightly asymmetric; costa single; mid-leaf with clear border of 3-5 rows of linear cells. Grows on bark of trees.

Specimens examined: Mymensingh: Phulpur, Jashim Sheikh, 18.3.1992; Netrokona: Netrokona Town, Aminul Haque, 13.10.1988; Tangail: Modhupur Forest, Farida Rahman, 12.3.1995; Ghatail, Farida Rahman, 12.3.1995.

Order : Hypnobryales; Family : Thuidiaceae

Genus : Thuidium B.S.G., Bryol. Eur. 5: 157 (1852).

37. Thuidium meyenianum (Hamp.) Doz. \& Molk., Bryol. Jav. 2: 121 (1865).

Hypnum meyenianum Hamp., Icon. Musc. 8: (1844).

Plants autoicous; delicate, wiry, very small, brownish-green forming mats, irregularly bipinnately branched; paraphyllia dense; leaves dimorphic; costa single; leaf cells obscure, papillose; seta papillose; capsule horizontal, gibbous; peristome normal, hypnoid. Grows on bark of trees.

Specimen examined: Mymensingh: Phulpur, Jashim Sheikh, 18.3.1975.

\section{Family : Amblystegiaceae}

Genus : Campylium ( Sull.) Mitt., J. Linn. Soc. Bot. 12: 631 (1863).

Hypnum Sect. Campylium Sull. Gray, Man. Bot. N.U. States, ed. 2: 677 (1856).

38. Campylium gollani C. Muell. ex Vohra, Bull. Bot. Surv. India 12(1-4): 101(1970).

Plants dioicous; small, glossy, golden-green; branches pinnate, dense; leaf slightly concave, apex acuminate; costa short, double, unequal; alar cells quadrate to subrectangular, sometimes inflated; middle leaf cells linear, apical cells rhomboid to linear; seta reddish; capsule brown. Grows on soil, often on stones.

Specimen examined: Mymensingh: Gouripur, Jashim Sheikh, 8.2.1993.

Family : Brachytheciaceae

Genus : Brachythecium B.S.G., Bryol. Eur. 6: 5 (1853).

39. Brachythecium curtum (Lindb.) Limpr., Laubm. Deutschl. 3: 101 (1896).

Specimen examined: Mymensingh: Ranikhony, S. Gomes, S. Naznin and Selina Banu, 26.5.1973. 
Family : Entodontaceae

Genus : Erythrodontium Hamp., Vid. Medd. Naturh.

For. Kjobenh. Ser. 3, 2: 279 (1870).

40. Erythrodontium julaceum (Schwaegr.) Par., Index. Bryol. 436 (1896).

Neckera julacea Hook. ex Schwaegr., Sp. Musc. Suppl. 3(1): 243 (1828).

Plants rigid, glossy, golden-green; closely pinnately branched; leaves terete, imbricate, closely appressed when dry; margin entire; costa absent; leaf cells elliptic to linear; alar conspicuous, rounded-quadrate. Grows on bark of trees.

Specimens examined: Jamalpur: Nakla, Abdus Sobhan, 13.7.1997; Islampur, Mokbul Hossain, 19.3.2000; Mymensingh: Khagdohor, Hasina Banu, 21.8.1996; Tangail: Mirzapur, Hamida Khatun, 15.3.1993.

\section{Family : Plagiotheciaceae}

Genus : Plagiothecium B.S.G. in Bryol. Eur. 5: 179 (1851).

41. Plagiothecium denticulatum (Hedw.) B.S.G., Bryol. Eur. 5: 190, 501 (1851).

Hypnum denticulatum L. ex Hedw. Sp. Musc.: 237 (1801).

Plants monoicous; robust, bright-green, glossy; leaves concave, asymmetric; costa double, often forked; leaf cells narrowly rhomboid, not papillose; alar cells absent. Grows on damp bricks.

Specimens examined: Jamalpur: Tarakandi, Md. Faruque, 7.9.1998; Madarganj, Abdus Sobhan, 24.11.1991; Kishoreganj: Kishoreganj Town, Fazlul Haque, 8.8.1998; Mymensingh: Teachers' Training College, Nazmul Hossain, 10.11.1988; Trishal, Khurshid Alam, 3.7.1977; Tangail: Bajitpur, Liakat Ali, 20.12.1976; Mirzapur, Hamida Khatun, 8.8.1999; Ghatail, Jaglul Pasha, 3.5.1997.

Genus : Stereophyllum Mitt., Ind. Or., 117 (1859).

42. Stereophyllum anceps (Bosch \& Lac.) Broth., Nat. Pfl. 1(3): 898 (1907).

Hypnum anceps Bosch \& Lac., in Bryol. Jav. 2: 161, 260 (1867).

Plants monoicous; light-green, silky, branches short, complanate; leaves asymmetrical; costa single, covers up to middle of leaf; leaf cells linear to rhomboid, smooth; seta erect, smooth; capsule inclined. Grows on bark of trees.

Specimens examined: Jamalpur: Circuit House area, Hamida Khatun, 2.12.1997; Jamalpur Town, Farida Rahman, 5.11.1997; Tarakandi, Md. Faruque, 7.9.1998; Mymensingh: Shaheb Para, Runu Dey, 9.12.1993; Muktagachha, Md. Fazlul Haque, 5.2.1994; Bangladesh Agriculture University, Shahana Nasreen, 2.8.1994; Fulbaria, Shahana Nasreen, 7.9.1994; Phulpur, Jashim Sheikh, 19.11.1997; Kazir Shimla, 
Rashiduddin, 19.1.1999; Netrokona: Netrokona Town, Hamida Khatun, 18.12.1999; Kendua, Md. Fazlul Haque, 18.7.1994; Mohanganj, Motaleb Hossain, 29.5.2000; Sherpur : Nakla, Abdus Sobhan, 7.9.1997; Tangail: Modhupur, Farida Rahman, 21.8.1996.

43. S. decorum (Mitt.) Wijk. \& Marg., in Taxon. 9: 52 (1960).

Hypnum decorum Mitt., in Musci Ind. Or. 77 (1859).

Plants autoicous; glossy, branches sparse and irregular; leaf cells smooth; costa strong covering 2/3 of leaf; seta slender, erect, smooth; capsule erect to slightly inclined; operculum long rostrate. Grows on bark of trees.

Specimens examined: Jamalpur: Nandina, Md. Fazar Ali, 3.2.1998; Islampur, Md. Fazle Rabbi, 15.6.1989 and Md. Abdus Sattar, 23.11.1998; Madarganj, Mukhlesur Rahman, 21.7.1999; Mymensingh, T.T. College, A.S.M. Asadur Rahman, 18.10.1997; Circuit House, K.B. Fattah, 10.10.1989; Sherpur: Rungtia Reserve Forest, Almas Uddin Hawlader, 13.12.1998; Tangail: Kagmari College, Kamruzzaman, 3.3.1987; Modhupur Forest, Farida Rahman, 12.6.1996; Kalihati, Sarwar Hossain, 12.3.1986.

44. S. ligulatum Jaeg., Ber. S. Gall. Naturw. Ges. (1877-78), 277, 1880.

Plants yellow-green, glossy; leaves concave and asymmetrical, ovate with obtuse apex; costa strong, single, covering about half of leaf length, upper cells rhomboid. Grows on damp brick walls and also on bark of trees.

Specimens examined: Jamalpur: Nandina, Md. Fazar Ali, 3.2.1988; Tarakandi, Md. Faruque, 7.9.1998; Islampur, Mokbul Hossain, 19.3.2000; Kishoreganj: Katiadi, Nahid Sultana, 20.12.1997; Tarail, S.M. Hannan, 7.9.1999; Karimganj, Mujibur Rahman, 8.7.1998; Mymensingh: Haluaghat, Md. Jashim Uddin, 1.19.89; Phulpur, Md. Abdul Awal, 4.5.1990; Durgapur, Md. Abdul Awal, 3.3.1994; Gaffargaon, Baroigaon, Md. Faisal Ahmed, 31.1.1998; T.T. College campus, Hamida Khatun, 15.11.1989; Bhaluka, Jamaluddin, 22.7.1997; Kazir Simla, Rashiduddin, 19.1.1999; Sherpur: Nakla, Monir Hossain, 12.12.1999; Sribordi, Asraf Hossain, 29.7.1998; Tangail : Dhanbari, Modhupur, Farida Rahman, 21.8.1886; Mirzapur, Hamida Khatun, 10.12.1995; Tangail Sadar, Akurtakur Para, S. Hadiuzzaman, 8.12.1996; Modhupur Forest, Farida Rahman, 24.6.1996; Rasulpur, Sakil Ahmed, 13.6.1997.

45. S. tavoyense (Hook.) Jaeg., Ber. S. Gall. Naturw. Ges. (1877-78), 279 (1880).

Hypnum tavoyense Hook., Icon. Pl. Rar. 1: 24 f.1 (1836).

Plants yellow-green, silky, lustrous; branches pinnate, complanate; leaves oblong with acute tip; costa single, ending in middle of leaf; alar distinct, quadrate; leaf cells smooth. Grows on bark of trees. 
Specimens examined: Jamalpur: Bakshiganj, Abdus Sattar, 5.2.1998; Tarakandi, Md. Faruque, 7.9.1998; Kishoreganj: Tarail, Rokonuddin, 7.9.1992; Karimganj, Mujibur Rahman, 8.7.1998; Mymensingh: T.T. College campus, Asadur Rahman, 18.10.1987 and Hamida Khatun, 15.11.1989; Ananda Mohan College, K.B. Fattah, 30.9.1989 and A.S.M. Asadur Rahman, 13.2.1993; Charkagia Kundu, Phulpur, Jesmin Akter, 20.8.1993; Muktagachha, Jesmin Akter, 20.8.1993; Trishal, Hamida Khatun, 28.6.1997; Gaffargaon, Hamida Khatun, 28.6.1997; Tarakandi, Saidul Islam, 23.12.1998; Kazir Simla, Rashiduddin, 19.1.1999; Sherpur: Gazni, Hamida Khatun, 28.11.1989; Sribordi, Asraf Hossain, 29.7.1998; Hatibandha, Abdus Sattar, 10.9.1998; Tangail: Modhupur Forest, Farida Rahman, 12.6.1995; Mirzapur, Hamida Khatun, 2.12.1995; Tangail Teacher’s Staff Quarter, S. Hadiuzzaman, 5.12.1996; Paharkanchanpur, Hamida Khatun, 3.4.1998.

\section{Family : Sematophyllaceae}

\section{Genus : Acroporium Mitt., J. Linn.Soc. Bot. 10: 182 (1868).}

46. Acroporium baviense (Besch.) Broth., Nat. Pfl. ed. 2, 11: 437 (1925).

Sematophyllum baviense Besch., J. de Bot. 4: 205 (1890).

Plants irregularly branched, loosely erect, spreading, golden-green; leaves concave, ovate-lanceolate, base auriculate, apex acute, sharp cuspidate, conspicuous alar tinted with about 5 curved, oblong, inflated cells at the angles. Grows on bark of trees.

Specimen examined: Tangail: Mirzapur, Motaleb Hossain, December 1998.

Genus : Taxithelium Spruce. ex Mitt., J. Linn. Soc. Bot. 12: 21, 496 (1869).

47. Taxithelium nepalense (Schwaegr.) Broth., Monsunia 1: 51 (1899).

Hypnum nepalense Schwaegr., Sp. Musc. Supple. 3(1): 226 (1828).

Plants autoicous; robust, yellow-green; branches irregular; leaves ovate with acute tips, concave; costa absent; leaf cells spindle-shaped with several small papillae in one row over the lumen; alar distinct, large rectangular; capsule inclined; peristome hypnoid. Grows on bark of trees.

Specimens examined: Jamalpur: Nandina, Fazar Ali, 3.2.1988; Islampur, Mokbul Hossain, 19.3.2000; Kishoreganj: Pakundia, Abdul Jabbar, 13.5.1991; Katiadi, Mojibur Rahman, 9.7.1999; Mymensingh: Phulpur, S. Hadiuzzaman, 24.9.1978 and Jashim Sheikh, 12.10.1998; Gaffargaon, Hamida Khatun, 3.11.1987; Haluaghat, Jashim Sheikh, 17.1.1993; Gouripur, Jashim Sheikh, 8.2.1993; Bangladesh Agriculture University campus, Shahana Nasrin, 18.8.1994; Koroitali, Shimanta Fari, Haluaghat, Shahana Nasrin, 21.12.1994; Gouripur, Md. Rafique, 12.2.1995; Gaffargaon, Faysal Ahmed, 28.6.1997; Churkhai, Naznin Ahmed, 28.6.1997; City Park, Hamida Khatun, 1.10.1997; 
Sherpur: Sribordi, Abu Sohel, 13.4.1998; Tangail: Dhanbari, Md. Kamruzzaman, 5.6.1987; Mirzapur, Nazrul Islam, 19.2.1991 and Hamida Khatun, 3.3.1992; Modhupur Forest, Farida Rahman, 12.6.1995; Shakhipur, Hamida Khatun, 22.8.1998 and Hassan Ahmed, 7.9.1998; Akurtakur Para, S. Hadiuzzaman, 5.12.1996; Chandra, Serajul Islam, 22.12.1998.

Family : Hypnaceae

Genus : Hypnum Hedw., Sp. Musc. 236 (1801).

48. Hypnum aduncoides (Brid.) C. Muell., Syn. 2: 295 (1851).

Hypnum cupressiforme Hedw. var.aduncoides Brid., Sp. Musc. 2: 219 (1812).

Plants robust, light-green, silky; branches irregularly pinnate; leaves erectopatent, falcate, concave; costa short, double; alar little differentiated; laminal cells linear. Grows on bark of trees.

Specimens examined : Mymensingh : Bangladesh Agriculture University, Lulu, Nasreen and Abdul Gani, April 1984; Netrokona : Kalmakanda, Al Mamun, 22.12.98;

Genus : Ectropothecium Mitt., J. Linn.Soc. Bot. 10: 180 (1868).

49. Ectropothecium perscabrum P. Tix., Rev. Bryol. Lichenol. 34: 171 (1966).

Plants robust, main stem creeping, branches pinnate; leaves erectopatent, falcate, asymmetric, concave, apex slightly dentate; leaf cells highly scabrous, narrow rhomboid; alar scarcely distinguished. Grows on bark of trees.

Specimen examined: Mymensingh: Trishal, Md. Monem and Md. Rafique, 12.12.1976.

Genus : Vesicularia (C. Muell.) C. Muell., Bot. Jahrb. 23: 330 (1896).

Hypnum Subsect. Vesicularia C. Muell., Syn. 2: 233 (1851).

50. Vesicularia dubyana (C. M.) Broth., E. \& P. Pflazenfam. ed.1 Musci, 1909.

Hypnum dubyanum C. M., Syn. 2, 241 (1851).

Plants autoicous; silky, in extensive mats; branches regularly pinnate; leaves broad, concave; costa short, double, often indistinct; leaf cells very lax, smooth; alar not differentiated; capsule pendulous, ovoid, large. Grows on bark of trees.

Specimen examined: Mymensingh: near Eidgah Maidan, K.B. Fattah, 10.10.1987.

51. V. reticulata (Doz. \& Molk.) Broth., Nat. Pfl. 1(3): 1094 (1908).

Hypnum reticulatum Doz. \& Molk., Ann. Sci. Nat. Bot. Ser. 3, 2: 309 (1844).

Plants autoicous; slender, glossy; branches irregularly pinnate; leaves broadly ovate: costa two, indistinct; alar absent; leaf cells smooth; capsule pendulous; peristome hypnoid. Grows on bark of trees. 
Specimens examined: Jamalpur: Islampur, Fazle Rabbi, 15.6.1989; Melananda, Golam Mostafa, 21.10.1999; Kishoreganj: Pakundia, Abdul Awal, 4.5.1990; Nandail, Abdul Awal, 4.5.1990; Mohera, Saidul Islam, 10.12.1988; Astagram, Motaleb Hossain, 18.9.1996; Circuit House, Jesmin Akter, 18.8.1992; Mymensingh: Durgapur, Farida Rahman, 4.1.1994; T.T. College campus, Nazmul Hossain, 10.11.1988 and K.B. Fattah, 10.12.1994; Ananda Mohan College campus, Asad Hossain, 26.10.1989; Bangladesh Agriculture University campus, Hamida Khatun, 9.11.1989; Ishwarganj, Hamida Khatun, 10.11.1989; Trishal, Hamida Khatun, 4.5.1996; Gaffargaon, Faysal Ahmed, 28.1.1997; Phulpur, Jashim Sheikh, 10.11.1997; Kazir Simla, Arif Ahmed, 28.6.1998; Sherpur: Kakla, Altaf Hossain, 27.9.2000; Tangail: Dhanbari, Md. Kamruzzaman, 5.6.1987; Ghatail, Farida Rahman, 10.8.1995; Modhupur Forest, Farida Rahman, 10.8.1995; Nagarpur, Md. Mohidur Rahman, 3.2.1996; Jamurki, Hamida Khatun, 3.4.1996; Akurtakur Para, S. Hadiuzzaman, 5.12.1996.

\section{References}

Banu, K. 1991. Taxonomic Studies on the Acrocarpous Mosses of Bangladesh. Ph.D. Thesis. Department of Botany, University of Dhaka, pp. 460.

Banu-Fattah, K. 1998. Bryophytic flora of Chittagong in Bangladesh. Bangladesh J. Plant Taxon. 5(2): 83-89.

Banu-Fattah, K. 2005. Funaria hygrometrica Hedw. (Funariaceae) from Bangladesh. Bangladesh J. Bot. 34(2): 121-124.

Banu-Fattah, K. and Hadiuzzaman, S. 1993. Taxonomic Studies on the Acrocarpous Mosses of Bangladesh. Three most widespread species. Plants for the environment. Proc. $7^{\text {th }}$ Bien. Bot. Conf. Bangladesh Bot. Soc. Dhaka, pp. 111-118.

Banu-Fattah, K. and Hadiuzzaman, S. 1994. Acrocarpous Mosses of Bangladesh-I. Family: Polytrichaceae. Bangladesh J. Plant Taxon. 1(1): 87-94.

Banu-Fattah, K. and Hadiuzzaman, S. 1995. Acrocarpous Mosses of Bangladesh-II. Family: Ditrichaceae. J. Asiat. Soc. Bangladesh. Sci. 21(2): 271-276.

Banu-Fattah, K. and Hadiuzzaman, S. 1996a. Acrocarpous Mosses of Bangladesh-III. Family: Dicranaceae. J. Asiat. Soc. Bangladesh, Sci. 22(1); 1-17.

Banu-Fattah, K. and Hadiuzzaman, S. 1996b. Acrocarpous Mosses of Bangladesh-IV. Family: Leucobryaceae. Bangladesh J. Plant Taxon. 3(1): 45-55.

Banu-Fattah, K. and Hadiuzzaman, S. 1996c. Acrocarpous Mosses of Bangladesh-VI. Family: Calymperaceae. Bangladesh J. Plant Taxon. 3(2): 59-76.

Banu-Fattah, K. and Hadiuzzaman, S. 1997. A new species of Splachnobryum C. Muell. (Splachnaceae) from Bangladesh. Bangladesh J. Bot. 26(1): 61-64.

Banu-Fattah, K. and Hadiuzzaman, S. 1998a. Acrocarppous Mosses of Bangladesh-V. Family: Fissidentaceae. J. Asiat. Soc. Bangladesh. Sci. 24(2): 215-249.

Banu-Fattah, K. and Hadiuzzaman, S. 1998b. Acrocarpous Mosses of Bangladesh-VII. Family: Pottiaceae. Bangladesh J. Plant Taxon. 5(2): 43-67.

Banu-Fattah, K. and Hadiuzzaman, S. 1998c. Acrocarpous Mosses of Bangladesh-VIII. Family: Funariaceae. Bangladesh J. Bot. 27(2): 71-87. 
Banu-Fattah, K. and Hadiuzzaman, S. 2003a. Acrocarpous Mosses of Bangladesh-IX. Family: Splachnaceae. Bangladesh J. Plant. Taxon. 10(1): 27-34.

Banu-Fattah, K. and Hadiuzzaman, S. 2003b. Acrocarpous Mosses of Bangladesh-X. Family: BryaceaeGenus Bryum Hedw. Bangladesh J Bot. 32(2): 107-117.

Banu-Fattah, K. and Hadiuzzaman, S. 2004. Acrocarpous Mosses of Bangladesh-X. Family: BryaceaeGenus Pohlia Hedw. Bangladesh J. Bot. 33(1): 31-34.

Banu-Fattah, K. and Hadiuzzaman, S. 2006a. Acrocarpous Mosses of Bangladesh-XI. Family: Bartramiaceae-1. Bangladesh J. Bot. 35(1):23-29.

Banu-Fattah, K. and Hadiuzzaman, S. 2006b. Acrocarpous Mosses of Bangladesh- XI. Family: Bartramiaceae-2. Bangladesh J. Bot. 35(2): 91-97.

Banu-Fattah, K. and Lal, J. 1998. Occurrence of Pleuridiella colei H.Robinson, a monotypic moss from Bangladesh. Bangladesh J. Bot. 27(2): 147-149.

Gangulee, H.C. 1969-1980. Mosses of Eastern India and adjacent regions. A Monograph. Vol 1-111 Books and Allied Pvt. Ltd., Calcutta, India, 2144 pp.

Hadiuzzaman, S. 1984. Mosses of Bangladesh II. A Taxonomic Study of Calymperes Sw. Bangladesh J. Bot. 13(2): 161-166.

Khatun, H. 2002. Taxonomic Studies on Pleurocarpic Mosses of Bangladesh. Ph.D. Thesis. Department of Botany, University of Dhaka, pp. 554.

Khatun, H. and Hadiuzzaman, S. 1994. Taxonomic Studies of some Pleurocarpic mosses of Bangladesh. Bangladesh J. Bot. 23(1): 113-122.

Khatun, H. and Hadiuzzaman, S. 1995. Addition to the Pleurocarpic mosses of Bangladesh. Bangladesh J. Bot. 24(2): 183-191.

Khatun, H. and Hadiuzzaman, S. 2003. Pleurocarpous mosses of Bangladesh. Family- Neckeraceae-I. Bangladesh J. Plant Taxon. 10(2): 47-55.

Khatun, H. and Hadiuzzaman, S. 2004. Pleurocarpous mosses of Bangladesh. Family- Erpodiaceae. Bangladesh J. Plant Taxon. 11(2): 29-32.

Khatun, H. and Hadiuzzaman, S. 2005. Pleurocarpous Mosses of Bangladesh: Meteoriaceae and Pterobryaceae. Bangladesh J. Plant Taxon. 12 (1): 53-57.

Khatun, H. and Hadiuzzaman, S. 2006. Pleurocarpous mosses of Bangladesh. Family- Entodontaceae. Bangladesh J. Plant Taxon. 13(2): 131-137.

Khatun, H. and Hadiuzzaman, S. 2007a. Pleurocarpous mosses of Bangladesh: Family- Sematophyllaceae. Bangladesh J. Bot. 36(1): 69-80.

Khatun, H. and Hadiuzzaman, S. 2007b. Additions to the Pleurocarpous mosses of Bangladesh: FamilyPlagiotheciaceae. Bangladesh J. Plant Taxon. 14(1): 79-82.

Robinson, H. 1964. A small collection of bryophytes from upper Assam, India. J. Hattori. Bot. Lab. 27: 124130.

Tixier, P. 1967. Bryophytae Indosinicae. The Dacca Univ. Stud. 25: 1-14. 